\title{
Get a look at Galleria
}

\author{
As researchers look for more mammalian alternatives, an old moth is learning new tricks.
}

\section{Jim Kling}

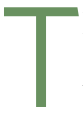
he Greater wax moth, Galleria mellonella, is a troublesome pest for beekeepers around the world. Also known as the honeycomb moth, its larvae burrow into honeycombs, leaving silklined tunnels that can entangle and injure the bees. But the larvae, easy to breed and maintain, have also been a boon to pet stores and tackle shops that sell them as food or fishing bait.

Since the 1980s, they have also shown great promise for infectious disease research - and, more recently, toxicology. As invertebrates, they have natural advantages over mice and rats for ethical reasons and compared to other spineless fellows such as the nematode or fruit fly, the moth larvae are larger - and thus easier to inject with test compounds - and can survive at human body temperature. That's crucial because many infectious agents express virulence genes only at this higher temperature.

And they are easy to work with. After arrival in the lab, they go straight into a refrigerator or incubator until they're ready for use, though with one caveat. "You can't leave them in a drawer for a week or they'll turn into moths, which we have had happen before," said Christopher Coates, an invertebrate immunologist at Swansea University in Wales.

Those properties have contributed to the moth's adoption as a model organism. For infectious disease research, researchers can inject lethal doses of bacteria followed by antimicrobial agents in an attempt to rescue the animals. Others have tested toxicity of various compounds, with general success - for example, in identifying the dose of an agent that kills half of the animals. These $\mathrm{LD}_{50}$ values are in agreement with those established in mammalian models.

These advantages have generated excitement in an era where regulatory agencies are seeking to reduce testing in mammalian models. "In the UK, when you look at regulations like the Animal Scientific Procedures Act, an insect larva can be considered a non-animal technology. You're removing a lot of the red tape, inspections of housing conditions, and you don't have to be specialized in training and handling or require expensive kit. They're a low maintenance alternative to using a

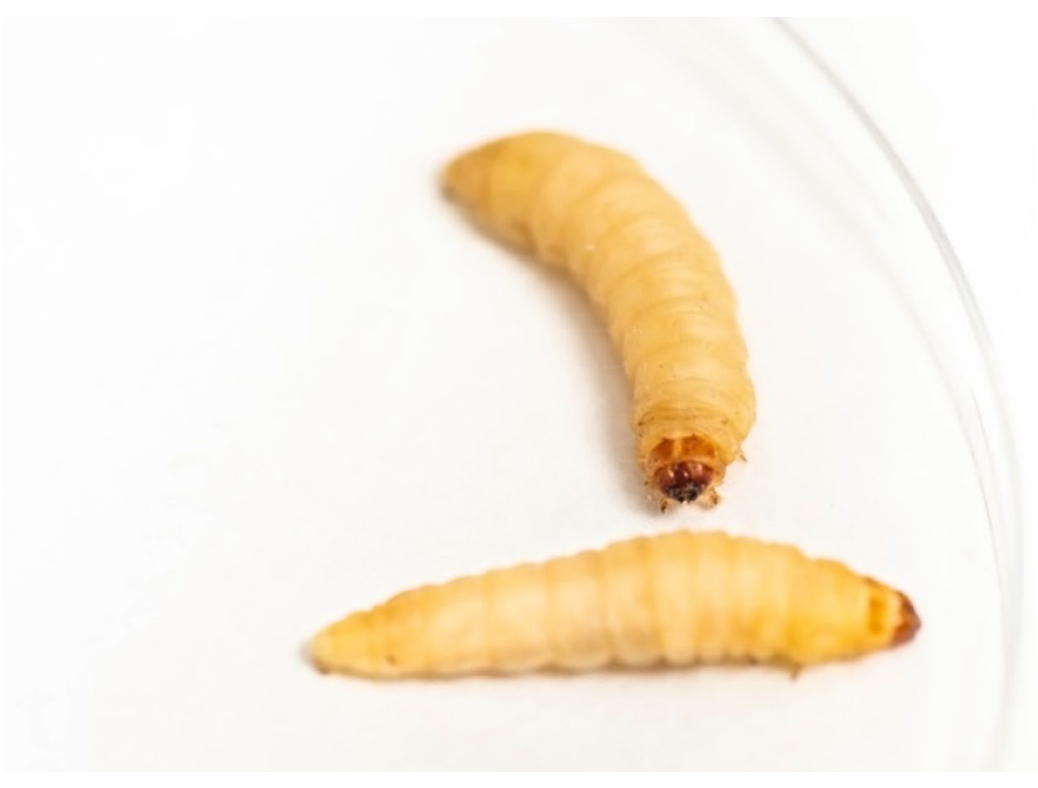

Not just bait | Galleria are a promising invertebrate model organism for a number of research areas. Credit: BioSystems Technology

mouse or a zebrafish, and having a highthroughput, cheap validated model that you can initially screen for all these indicators of potency is very attractive, especially if you have thousands of compounds, or thousands of strains of microbes," said Coates.

But despite its general utility, Galleria has serious limitations. It lacks many of the complex organ systems found in mammals, and like all other invertebrates, the larvae have no adaptive immune system, though the innate system has proven to share similarity with humans ${ }^{1}$. But even there, differences abound. "We constantly use the expression that Galleria shares the innate immune response of vertebrates, and that's true to a certain extent. There are phagocytes in Galleria and humans and mice, and we're finding a lot of conserved mechanisms. But it sort of stops there.

There hasn't been much progress lately on the molecular and cellular mechanisms of immunity that are similar between Galleria and murine models," said Coates.

Such research however has gained traction with the publication of the Galleria genome in $2018^{2}$, including the mitochondrial genome 3 . "It's a major step forward," said Coates.

\section{The Same, but different}

Comparative studies have shown that Galleria has plenty in common with mammals; this includes conserved pathogen recognition mechanisms, or at least functional analogs that lead to similar symptoms when disrupted in organs like the gut". "For general purpose screening, the jury is in: We know it's a really good alternative. Biochemical labs, companies, they've adopted this as high throughput and proof-of-concept (screens) for compounds and pharmaceuticals," said Coates. That allows them to triage compounds and conduct fewer studies in mice.

But Coates' group wants to expand the model's use. One area of key interest is gut toxicity and gastric ulceration that can occur with non-steroidal anti-inflammatory drugs (NSAIDs) and other compounds. That work is currently done by testing for gut 'leakiness' in rats. To determine if Galleria could be used as a substitute, the team inoculated larvae with indomethacin, 
an NSAID commonly used in assays to identify novel compounds with restorative properties. They injected the molecule into the mid-gut of the larvae, which has architectural similarities to the human intestines, and then monitored immune cell numbers and changes in gut permeability.

The team followed up with histopathology screening, X-ray microtomography/microscopy, and enzyme assays to detect changes after exposure. There were increases in circulating immune cells, while fluorescent microspheres revealed more gut leakiness. There was also evidence of tissue damage in the midgut, such as cellular necrosis and epithelial sloughing, along with heightened detoxification activity ${ }^{5}$.

"In regions of tissue that mimic most closely the human GI tract, we see very similar symptoms. We propose that you can use those insects at least as a first round triage to narrow compounds that potentially have gut disturbing properties, and with this focus you can reduce the number of mice needed, and you can complement that work with cell culture," said Coates.

Kevin Kavanagh, a biologist at Maynooth University in Ireland, has worked with Galleria for two decades. His team has examined how the larvae metabolize various compounds. Insects don't have livers, the organ where most human metabolism occurs, but rather a structure called the fat body that performs similar functions. In separate studies of caffeine and potassium nitrate, Kavanagh's team showed that they can isolate metabolites in the moth larvae that correspond well to those seen in mouse models. "That should give you a good indication of the likely effect in mammals," said Kavanagh.

In the potassium nitrate study, his group demonstrated that the $\mathrm{LD}_{50}$ value obtained in Galleria is comparable to the value found in rats $^{6}$. He recently helped one company narrow a field of 70 antibacterial drug candidates down to 15 that they eventually took into mouse studies, saving considerable time and expense. "We get results in 24 or 48 hours, where a lot of murine work takes weeks," said Kavanagh.

\section{New pathogen studies}

Although Galleria has long been a model for screening different compounds, researchers continue to expand its use. Jacelyn Loh, a senior research fellow at the University of Auckland, is using Galleria to study virulence factors in Group A Streptococcus (GAS) bacteria. Her team is investigating thin, hair-like structures called pili that protrude from the surfaces of the bacterial

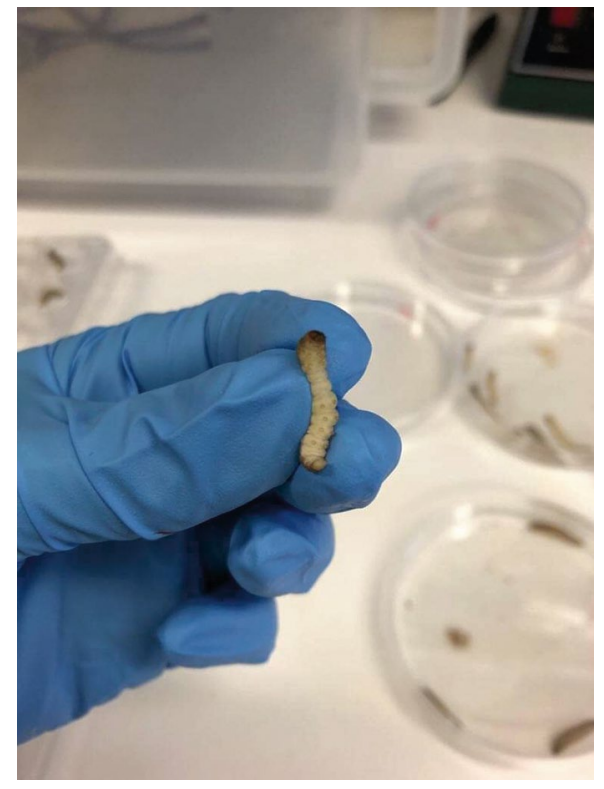

Larvae in the hand I The moths' larvae grow well at huma-relevant temperatures and are large and easy to manipulate. Credit: $\mathrm{H}$. Aghababa

cell, and whether the proteins found in them could be good vaccine candidates.

They are now searching for GAS pathogenesis genes. With a candidate in hand, they can create a GAS knockout but need to test it in an animal model to determine if the bacteria's pathogenicity has been affected. Unfortunately, because GAS naturally infects only humans, attempting to study GAS in any model requires unnaturally high doses. "However, animal and insect models provide us with valuable tools to indicate what may happen in humans. We can use Galleria quite effectively as a screening tool to give us an indication that we should proceed with more laborious techniques used in animals. We try lots of different tests to confirm the same thing," said Loh.

But Galleria, like all invertebrates, lacks an adaptive immune system and cannot produce antibodies in response to an inoculation. Should a candidate vaccine emerge from the moth screens, the group will turn to a vertebrate model, whether rats or mice, to test its efficacy. "

Galleria is also finding use in microbiome work. Like rodents, Galleria's microbiome differs from that of humans, but it has the virtue of being quite simple: its microbiome is dominated by Enterococcus bacteria. Its lack of an adaptive immune system, a hindrance in other research areas, can be a bonus here. It allows researchers to examine how a probiotic and a pathogen interact, with little interference from an existing microbiota or the immune system, according to Sarah Lebeer, a microbiologist at the University of Antwerp.

Lebeer's team is developing next generation probiotics that they have isolated from the human microbiome and plan to test them in Galleria to help ensure that they are safe. She notes that human pathogens such as Staphylococcus aureus and Pseudomonas aeruginosa will kill Galleria, whereas all known beneficial bacteria tested to date have proven safe in the moths. "It's a good indication if your bacteria have virulence factors," said Lebeer.

\section{Setting new standards}

Galleria has some catching up to do in at least one important area. Until recently, it wasn't possible to buy larvae that have been raised under controlled conditions. Instead, researchers had to go to their local pet shop or bait store, and that introduces problematic variability into experimental systems, according to Olivia Champion. "You wouldn't think of using a mouse you found in your pantry or bought from the pet shop for your research experiments," said Champion, who is co-founder and CEO of Biosystems Technology in the UK, a vendor of Galleria larvae standardized for scientific use.

Champion's own research began with mammalian models, when she was working with food poisoning-associated Campylobacter bacteria. Mice however didn't work - they aren't susceptible enough to infection. Frustrated, she read a couple of papers on Galleria and took a chance.

"To my amazement I could see a really clear response in the larvae. The high-dose group all died, and I could work out the $\mathrm{LD}_{50}$. That opened a whole avenue of research that I had wanted to carry out on Campylobacter during my post-doc," said Champion. Still, there was a problem. "There was lots of noise in the system. The error bars were quite large," she says. She decided to standardize the model in hopes of making outcomes more predictable.

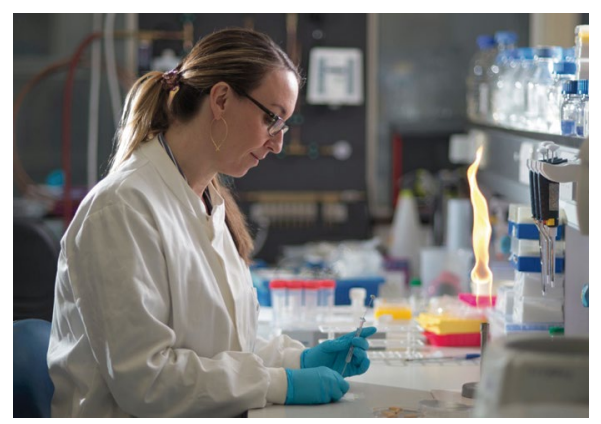

Olivia Champion. Credit: BioSystems Technology 


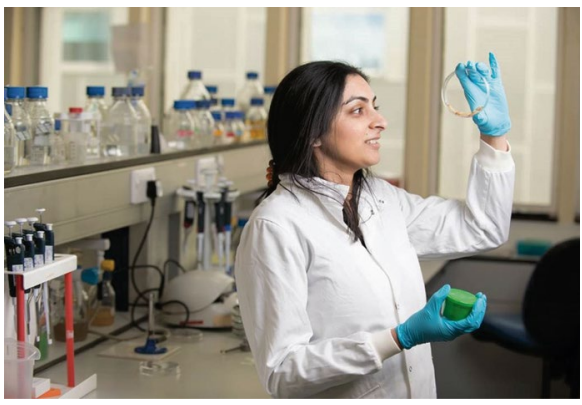

Sariqa Wagley. Credit: BioSystems Technology

Champion suspected that the conditions larvae were raised under might be affecting the results, so she investigated and found that colonies were often treated with antibiotics to control infections as well as hormones to extend the larval stage. That means that researchers typically can't know if the larvae they use have been exposed to antibiotics, or what type - a complication particularly important for those testing antibiotics, pathogenicity, or microbiota. To make matters worse, Galleria bred at different facilities, especially in different countries, could be genetically distinct.

Champion set up a standardized line of larvae for her own use, but a telling test came when a postdoc colleague named Sariqa Wagley at the University of Exeter, where Champion worked at the time, also found herself struggling to use Galleria to identify a virulence gene in Vibrio parahaemolyticus, a marine bacteria that can cause gastroenteritis after eating shellfish. She had found a strain of the bacteria that was infectious to humans, but it didn't contain any known toxins. She sequenced the genome and identified a potential novel virulence gene called MutT, then knocked it out and attempted to show that more larvae survived than those infected with the wild type.

More of the larvae survived when infected with the mutant bacteria, but the system was noisy and the results didn't reach statistical significance. "She could never demonstrate a phenotype using these bait shop larvae. She repeated the studies over and over for six months, and kept getting what she thought was a (positive result), but she couldn't prove it," said Champion.

Champion had just finished up her controlled system - which she called TruLarv - and suggested that Wagley try them. The results were immediate. "When I used TruLarv in my experiments the results were consistent and reproducible and showed that $V$. parahaemolyticus MutT gene was a virulence factor," recalls Wagley. She went on to publish the results in the journal Virulence ${ }^{7}$.

The cost is higher than pet shop larvae - at about 50 pounds for 50 larvae — but the research benefits can make it worth it, said Champion. "It's not just the cost of your reagent, it's the time taken," she said, noting the time that Wagley had devoted to tantalizing but inconclusive results.

\section{Convincing the influencers}

Despite the promise of Galleria, challenges remain. Funders and regulators need to be convinced of its utility. Regulatory agencies remain especially skeptical, according to Coates. "The concept of going from a mouse to an insect is still drastic to people. But we're trying to demystify it," he said.
The 2016 publication of the Galleria genome holds promise for the generation of human disease models, such as mutants that mimic cystic fibrosis or other human diseases. Other researchers see the potential to better understand the immune system and its interaction with pathogens. "To be able to genetically manipulate Galleria would be a quite a big step forward in the field, so that we can for example label or even knock out certain cell types and see how the larvae function without them," said Loh.

That vision hasn't come to fruition yet, though there is optimism. "A large number of groups are looking to start knocking out genes, but to the best of my knowledge there hasn't been much success just yet," said Kavanagh. He cited no technical barrier, just a lack of funding: "It's a perennial story for research - trying to convince funders to support research for a particular project that they might not see the immediate value of. That's an ongoing challenge."

Jim Kling

Freelance science writer, Bellingham, WA, USA.

e-mail: jkling@gmail.com

Published online: 25 February 2020

https://doi.org/10.1038/s41684-020-0489-1

\section{References}

1. Browne, N., Heelan, M. \& Kavanaugh, K. Virulence 4, 597-603 (2013).

2. Lange, A. et al. Genome Announc 6, e01220-17 (2018).

3. Park, Y. et al. Mitochondrial DNA B 2, 714-715 (2018).

4. Lange, A. et al. Front Immunol 9, 2114 (2018).

5. Emery, H., Johnston, R., Rowley, A. F. \& Coates, C. J. Arch Toxicol 93, 2347-2360 (2019).

6. Maguire, R., Duggan, O. \& Kavanaugh, K. Cell Biol Toxicol 32, 209-216 (2016).

7. Wagley, S. et al. Virulence 9, 197-207 (2018). 